\title{
Higher signal harmonics, LISA's angular resolution, and dark energy
}

\author{
K. G. Arun, ${ }^{1,2, *}$ Bala R. Iyer, ${ }^{3, \dagger}$ B. S. Sathyaprakash, ${ }^{4, \$}$ Siddhartha Sinha, ${ }^{3,5, \S}$ and Chris Van Den Broeck ${ }^{4, \|}$ \\ ${ }^{1}$ LAL, Université Paris-Sud, IN2P3/CNRS, Orsay, France \\ ${ }^{2} G \mathbb{R e} \mathbb{C O}$, Institut d'Astrophysique de Paris-C.N.R.S., Paris, France \\ ${ }^{3}$ Raman Research Institute, Bangalore, 560 080, India \\ ${ }^{4}$ School of Physics and Astronomy, Cardiff University, 5, The Parade, Cardiff, United Kingdom, CF24 $3 Y B$ \\ ${ }^{5}$ Department of Physics, Indian Institute of Science, Bangalore, 560 012, India \\ (Received 27 July 2007; published 9 November 2007; publisher error corrected 21 December 2007)
}

It is generally believed that the angular resolution of the Laser Interferometer Space Antenna (LISA) for binary supermassive black holes (SMBH) will not be good enough to identify the host galaxy or galaxy cluster. This conclusion, based on using only the dominant harmonic of the binary SMBH signal, changes substantially when higher signal harmonics are included in assessing the parameter estimation problem. We show that in a subset of the source parameter space the angular resolution increases by more than a factor of 10, thereby making it possible for LISA to identify the host galaxy/galaxy cluster. Thus, LISA's observation of certain binary SMBH coalescence events could constrain the dark energy equation of state to within a few percent, comparable to the level expected from other dark energy missions.

DOI: 10.1103/PhysRevD.76.104016

\section{INTRODUCTION}

An outstanding issue in present day cosmology is the physical origin of dark energy (see, e.g., Ref. [1] for a review). Probing the equation-of-state ratio $[w(z)]$ provides an important clue to the question of whether dark energy is truly a cosmological constant (i.e., $w=-1$ ). Assuming the Universe to be spatially flat, a combination of Wilkinson Microwave Anisotropy Probe (WMAP) and Supernova Legacy Survey (SNLS) data yields significant constraints on $w=-0.967_{-0.072}^{+0.073}$ [2]. Without including the spatial flatness as a prior into the analysis, WMAP, large-scale structure, and supernova data place a stringent constraint on the dark energy equation of state, $w=$ $-1.08 \pm 0.12$. The Laser Interferometer Space Antenna (LISA) could play an important role in investigating the nature of dark energy as argued in Refs. [3,4].

Binary supermassive black holes (SMBH), often referred to as gravitational-wave (GW) "standard sirens" (analogous to the electromagnetic "standard candles") [5], are potential sources for the planned LISA mission. LISA would be able to measure the "redshifted" masses of the component black holes and the luminosity distance to the source with good accuracy for sources up to redshifts of a few. However, GW observations alone cannot provide any information about the redshift of the source. If the host galaxy or galaxy cluster is known, one can disentangle the redshift from the masses by optical measurement of the redshift. This would not only allow one to extract the "physical" masses, but also provide an exciting possibility to study the luminosity distance-redshift relation providing

\footnotetext{
*arun@lal.in2p3.fr

†bri@rri.res.in

\#B.Sathyaprakash@astro.cf.ac.uk

${ }^{\S}$ p_siddhartha@ rri.res.in

"Chris.van-den-Broeck@astro.cf.ac.uk
}

PACS numbers: 04.30.Db, 04.25.Nx, 04.80.Nn, 95.55.Ym

a totally independent confirmation of the cosmological parameters. Further, this combined observation can be used to map the distribution of black hole masses as a function of redshift [6-8]. For this to be possible, LISA should (a) measure the luminosity distance to the source with a good accuracy and (b) localize the coalescence event on the sky with good angular resolution so that the host galaxy/galaxy cluster can be uniquely identified.

References [3,4] identified two potential problems in using binary SMBH as standard sirens. First, they found that LISA's angular resolution might not be good enough to identify the source galaxy or galaxy cluster, and that other forms of identification would be needed, and second, they pointed out that weak lensing effects would corrupt the distance estimation to the same level as LISA's systematic error on the measurement of the luminosity distance. Their analyses, like most others in the literature on LISA parameter estimation, were based on the so-called restricted post-Newtonian (PN) waveforms. The restricted waveforms (RWF) retain only the leading order (i.e., Newtonian) term in the wave amplitude, a PN series, but incorporate the phase up to the maximum available PN order, which is currently 3.5PN [9-12]. Recent studies have shown that the inclusion of higher order amplitude terms in the waveform (and hence higher harmonics of the orbital frequency) would play an important role in the detection rates (by increasing the mass reach of the detector) [13-15] as well as in the problem of parameter estimation [16-20] of both ground-based and space-based detectors. Specifically, Refs. [18,19] examined the improved angular resolution of different space-based detector configurations due to the inclusion of higher harmonics.

In the present work, we revisit the problem of parameter estimation in the context of LISA using amplitudecorrected PN waveforms. We investigate systematically the variation in parameter estimation with PN orders by critically examining the role of higher harmonics in the fast 
GW phasing, higher PN corrections in the amplitudes and frequency sweep, and their interplay with the slow modulations induced due to LISA's motion. More importantly, we explore the improvement in the estimation of the luminosity distance and the angular parameters due to the inclusion of higher harmonics in the waveform. We translate the error in the angular resolution to obtain the number of galaxies (or galaxy clusters) within the error box on the sky. We find that, independent of the angular position of the source on the sky, higher harmonics improve LISA's performance on both counts raised in Refs. [3,4]: On the one hand, we will show that the angular resolution improves typically by a factor of $\sim 2-500$ (greater at higher masses) and the error on the estimation of the luminosity distance goes down by a factor of $\sim 2-100$ (again, larger at higher masses). For many possible sky positions and orientations of the source, the inaccuracy in our measurement of the dark energy would be at the level of a few percent, so that it would only be limited by weak lensing. We conclude that LISA could provide interesting constraints on cosmological parameters, especially the dark energy equation of state, and yet circumvent all the lower rungs of the cosmic distance ladder.

This paper is structured as follows. In the next section we introduce our signal model and the LISA noise power spectral density (PSD) we will use. In Sec. III we discuss our results on parameter estimation and their relevance for astrophysics and cosmology. Section IV gives an overview of various effects that are likely to affect our estimates. Conclusions are presented in Sec. V. Technical details on how the parameter estimation was performed can be found in Appendix A. Finally, in Appendix B we give an in-depth discussion of the way parameter estimation is influenced by the inclusion of higher harmonics and their amplitude corrections.

\section{SIGNAL MODEL AND LISA NOISE PSD}

The post-Newtonian formalism has been used to study the evolution of a binary under gravitational radiation reaction to a very high order in the small parameter $v$ characterizing the velocity of the component objects, yielding accurate expressions for the orbital phase and the two gravitational-wave polarizations. For binaries consisting of component stars of negligible spin on quasicircular orbits, the most accurate computations currently known have corrections not only to the orbital phase up to order $v^{7}$ (i.e., 3.5PN order in the notation of PN theory) $[9,11,12,21,22]$, but also corrections to the gravitationalwave polarizations to order $v^{5}$ (i.e., 2.5PN order) [23-25]. We shall call this the "full" waveform (FWF).

The waveform as seen in LISA is modulated in two ways due to LISA's motion. LISA consists of three spacecraft at the vertices of an equilateral triangle of $5 \times 10^{6}$ kilometers, each craft on a heliocentric orbit slightly inclined to the ecliptic. As the craft orbit the sun, the triangular formation also spins around itself with the same one-year period as the orbital period. Therefore, relative to LISA the source location and orientation changes with time with a one-year period and induces amplitude and phase modulations in the waveform.

It is well known that, at signal frequencies $f \lessgtr$ $5 \times 10^{-3} \mathrm{~Hz}$, LISA can essentially be modeled as a pair of two-arm interferometers, usually labeled as I and II [26], and this suffices for the sources considered in this paper. (However, it would be interesting to investigate the added value of the remaining third combination ignored in this work.) In what follows, to begin with, we consider a single detector.

Let us consider a source of total mass $M=m_{1}+m_{2}$ and symmetric mass ratio $\nu=m_{1} m_{2} / M^{2}$ (where $m_{1}, m_{2}$ are the individual component masses) located at a luminosity distance $D_{\mathrm{L}}$. In the stationary phase approximation (SPA), the Fourier transform $\tilde{h}_{\mathrm{I}}(f)$ of the response of detector I to the full waveform, including the modulations due to LISA's motion, is given by [15]

$$
\tilde{h}_{\mathrm{I}}(f)=\frac{\sqrt{3}}{2} \frac{2 M \nu}{D_{L}} \sum_{k=1}^{7} \sum_{n=0}^{5} \frac{A_{(k, n / 2)}^{\mathrm{I}}\left(t\left(f_{k}\right)\right) x^{(n / 2)+1}\left(t\left(f_{k}\right)\right) e^{-i \phi_{(k, n / 2)}^{\mathrm{I}}\left(t\left(f_{k}\right)\right)}}{2 \sqrt{k \dot{F}\left(t\left(f_{k}\right)\right)}} \exp \left[i \psi_{f, k}\left(t\left(f_{k}\right)\right)\right]
$$

where $f_{k} \equiv f / k$, an overdot denotes derivative with respect to time, and $\psi_{f, k}\left(t\left(f_{k}\right)\right)$ is given by

$\psi_{f, k}\left(t\left(f_{k}\right)\right)=2 \pi f t\left(f_{k}\right)-k \Psi\left(t\left(f_{k}\right)\right)-k \phi_{\mathrm{D}}\left(t\left(f_{k}\right)\right)-\pi / 4$.

In Eq. (2.1), the factor $\sqrt{3} / 2$ is due to the $60^{\circ}$ angle between the interferometer's arms, and $t(f)$ is given in Ref. [11]. The waveform is a superposition of harmonics of the orbital frequency (labeled by the index $k$ ), and each harmonic has PN contributions to the amplitude (labeled by $n$; note that we can only go up to $n=5$, as no amplitude corrections are explicitly known beyond 2.5PN). As the PN order in amplitude is increased, more and more harmonics appear; at 2.5PN order there are seven, which is why the index $k$ only runs up to $k=7$. Quantities in Eqs. (2.1) and (2.2) with the argument $t\left(f_{k}\right)$ denote their values at the time when the instantaneous orbital frequency sweeps past the value $f / k$, and $x(t)$ is the PN parameter given by $x(t)=$ $(2 \pi M F(t))^{2 / 3}, \quad F(t)$ being the instantaneous orbital 
frequency of the binary. $A_{(k, n / 2)}^{\mathrm{I}}(t)$ and $\phi_{(k, n / 2)}^{\mathrm{I}}(t)$ are the polarization amplitudes and phases of the $k$ th harmonic appearing at the $n / 2$ th PN order. $\Psi(t)$ is the orbital phase of the binary and $\phi_{\mathrm{D}}(t)$ is a time-dependent term representing Doppler modulation. Explicit expressions for $A_{(k, n / 2)}^{\mathrm{I}}$ and $\phi_{(k, n / 2)}^{\mathrm{I}}$ can be found in [14]; time dependence of these quantities arises through the beam-pattern functions due to the varying sky position and orientation of the source relative to the detector [26]. The expression for $\phi_{\mathrm{D}}(t)$ is given in [15,26]. For the PN expansions for $t(F), \Psi(F)$, $\dot{F}(F)$, we refer to Ref. [11].

The RWF corresponds to retaining the term with $k=2$ and $n=0$ in Eq. (2.1) and neglecting all others. It is clear that the RWF has only the dominant harmonic at twice the orbital frequency but no other harmonic, nor PN corrections to the dominant one. It does, however, include the post-Newtonian expansion of the phase to all known orders, i.e., up to $v^{7}$. The FWF, on the other hand, not only has the dominant harmonic but also other harmonics up to 7 times the orbital frequency and their PN corrections to the relevant order. The distinctive nature of the FWF as compared to RWF, especially the richer structure in its spectrum, can be seen in Fig. 1 of Refs. [15,20].

Expanding the denominator and extracting the lowest order term helps us rewrite the waveform in a form more suitable for our purposes,

$$
\begin{aligned}
\tilde{h}_{\mathrm{I}}(f)= & \frac{\sqrt{5}}{8} \frac{1}{\pi^{2 / 3}} \frac{\mathcal{M}^{5 / 6}}{D_{\mathrm{L}}} \frac{1}{(2 f)^{7 / 6}} \sum_{k=1}^{7} k^{2 / 3} e^{i \psi_{f, k}\left(t\left(f_{k}\right)\right)} \\
& \times\left[\left(\sum_{n=0}^{5} A_{(k, n / 2)}^{\mathrm{I}}\left(t\left(f_{k}\right)\right)\left(2 \pi M f_{k}\right)^{n / 3} e^{-i \phi_{(k, n / 2)}^{\mathrm{I}}\left(t\left(f_{k}\right)\right)}\right)\right. \\
& \left.\times\left(\sum_{m=0}^{5} S_{(m / 2)}\left(2 \pi M f_{k}\right)^{m / 3}\right)\right]_{p}
\end{aligned}
$$

where $\mathcal{M}=M \nu^{3 / 5}$ is the chirp mass of the binary, and $[\cdots]_{p}$ denotes consistent truncation to $p$ th post-Newtonian order (in our case $p=2.5$ ). The coefficients $S_{(m / 2)}$ are the PN expansion coefficients of $\dot{F}\left(t\left(f_{k}\right)\right)^{-1 / 2}$ and are given in Eq. (A.4) of [14].

Each harmonic in $\tilde{h}_{\mathrm{I}}(f)$ is taken to be zero outside a certain frequency range. The upper cutoff frequencies are dictated by the last stable orbit (LSO), beyond which the PN approximation breaks down. For simplicity we assume that this occurs when the orbital frequency $F(t)$ reaches $F_{\text {LSO }}=1 /\left(6^{3 / 2} 2 \pi M\right)$ - the orbital frequency at LSO of a test particle in Schwarzschild geometry in $c=G=1$ units. ${ }^{1}$ Consequently, in the frequency domain, the contribution to $\tilde{h}(f)$ from the $k$ th harmonic is set to zero for frequencies above $k F_{\mathrm{LSO}}$. In determining the lower cutoff

\footnotetext{
${ }^{1}$ Note that the cutoffs are placed on the orbital frequency of the binary, not the dominant GW harmonic, hence the extra factor of 2 in the denominator of the expression for $F_{\mathrm{LSO}}$.
}

frequencies we assume that the source is observed for at most one year, and the $k$ th harmonic is truncated below a frequency $k F_{\text {in }}$, where $F_{\text {in }}$ is the value of the orbital frequency one year before LSO is reached [15]:

$$
F_{\text {in }}=F\left(t_{\text {LSO }}-\Delta t_{\text {obs }}\right)=\frac{F_{\text {LSO }}}{\left(1+\frac{256 \nu}{5 M} \Delta t_{\mathrm{obs}} v_{\mathrm{LSO}}^{8}\right)^{3 / 8}} .
$$

For simplicity the quadrupole formula was used. In the above, $t_{\text {LSO }}$ and $v_{\text {LSO }}=1 / \sqrt{6}$ are, respectively, the time and orbital velocity at last stable orbit, and $\Delta t_{\mathrm{obs}}=1 \mathrm{yr}$. However, LISA's sensitivity becomes poorer and poorer below $\sim 3 \mathrm{mHz}$ and current estimates normally assume a "noise wall" at $f_{\mathrm{s}}=10^{-4} \mathrm{~Hz}$. Thus, we take the lower cutoff frequency of the $k$ th harmonic to be the maximum of $f_{\mathrm{s}}$ and $k F_{\text {in. }}$. For more details we refer to [15].

As we mentioned before, the LISA detector can be viewed as a combination of two independent detectors. Until now we have dealt with one detector. Calculations for the two detector case closely follow the corresponding treatment for the RWF, which is explained in detail in [26]. The beam-pattern functions for detector II can be obtained from those of detector I by a simple rotation. The waveform $\tilde{h}_{\mathrm{II}}(f)$ for the second detector is formally identical to $\tilde{h}_{\mathrm{I}}(f)$, and quantities $A_{(k, n / 2)}^{\mathrm{II}}$ and $\phi_{(k, n / 2)}^{\mathrm{II}}$ are obtained from their counterparts $A_{(k, n / 2)}^{\mathrm{I}}$ and $\phi_{(k, n / 2)}^{\mathrm{I}}$ by replacing the beam-pattern functions of detector I by those of detector II.

The waveform given in Eq. (2.3) together with its counterpart for the second detector form the basis of the analysis in this paper. Following earlier works of Refs. [6,7,26$28]$ we employ the Fisher matrix approach $[29,30]$ to the problem of parameter estimation. The waveforms depend on nine parameters which are chosen to be

$$
\mathbf{p} \equiv\left(\ln \mathcal{M}, \delta, t_{\mathrm{C}}, \phi_{\mathrm{C}}, \ln D_{\mathrm{L}}, \mu_{\mathrm{S}}, \mu_{\mathrm{L}}, \phi_{\mathrm{S}}, \phi_{\mathrm{L}}\right),
$$

where $\delta \equiv\left(m_{2}-m_{1}\right) / M\left(m_{1}\right.$ and $m_{2}$ being the individual masses; throughout this paper we assume $\left.m_{2} \geq m_{1}\right)^{2}$ is used as a parameter instead of $\nu$, following Ref. [20]; $t_{\mathrm{C}}$, $\phi_{\mathrm{C}}$ are, respectively, the time and orbital phase at coalescence $^{3} ; \mu_{\mathrm{S}}=\cos \theta_{\mathrm{S}}$ and $\phi_{\mathrm{S}}$ determine the source position in the sky; while $\mu_{\mathrm{L}}=\cos \theta_{\mathrm{L}}$ and $\phi_{\mathrm{L}}$ determine the orientation of the binary's orbit with respect to a nonrotating

\footnotetext{
${ }^{2}$ The waveform is invariant under interchange of mass labels provided that, at the same time, the phasing is shifted by $\pi$; since we use a single phasing formula we need an ordering in the definition of $\delta$. The parameter $\delta$ was originally introduced in place of $\nu$ to ensure that the FWF Fisher matrix remains nonsingular on the surface $m_{1}=m_{2}$. The situation is reversed in the case of RWF: there the Fisher matrix becomes singular in the limit $\delta \rightarrow 0$ whereas it remains regular at $m_{1}=m_{2}$ when $\nu$ is used in place of $\delta$ (see the discussion in [20]). The equal mass case is dealt with in more detail in [31].

${ }^{3}$ Below we will consistently set the values of $t_{\mathrm{C}}$ and $\phi_{\mathrm{C}}$ to zero, but both parameters are included as coordinates on the space of signals in computing the Fisher matrix.
} 
detector at the solar-system barycenter. ${ }^{4}$ Following Ref. [26], we have fixed the initial position and orientation of LISA by setting the constants $\phi_{0}$ and $\alpha_{0}$ defined there to zero at $t=0$. The polarization amplitudes and phases depend on the location and orientation of the source through the beam-pattern functions. The orientation of the source changes relative to LISA with the period of a year. Therefore, these quantities are modulated on a oneyear time scale and depend on $\mu_{\mathrm{S}}, \mu_{\mathrm{L}}, \phi_{\mathrm{S}}, \phi_{\mathrm{L}}$ and also on $\delta, \ln \mathcal{M}$, and $t_{\mathrm{C}}$. The phase $\psi_{f, k}\left(t\left(f_{k}\right)\right)$ depends on $\ln \mathcal{M}, \delta$, $t_{\mathrm{C}}$, and $\phi_{\mathrm{C}}$ and varies with the orbital time scale which is much shorter than a year.

Following Ref. [32], the parameters can be subdivided into two subcategories: $\mathbf{p}_{\text {fast }}=\left(\ln \mathcal{M}, \delta, t_{\mathrm{C}}, \phi_{\mathrm{C}}\right)$ and $\mathbf{p}_{\text {slow }}=\left(\ln D_{\mathrm{L}}, \mu_{\mathrm{S}}, \mu_{\mathrm{L}}, \phi_{\mathrm{S}}, \phi_{\mathrm{L}}\right)$. The classification arises naturally because the signal that LISA observes can be viewed as a (slow) low frequency modulation due to its motion around the sun superposed on the (fast) high (GW) frequency carrier signal. The accuracy of estimation of $\mathbf{p}_{\text {fast }}$ follows from the GW phasing of the binary while that of $\mathbf{p}_{\text {slow }}$ from the modulations associated with LISA's orbital motion.

In our analysis, we take the noise power spectral density (PSD) to be that given by Eqs. (2.28)-(2.32) of Ref. [7]. As usual, the Fisher matrix $\Gamma$ for LISA as a whole is simply $\Gamma=\Gamma_{\mathrm{I}}+\Gamma_{\mathrm{II}}$, where $\Gamma_{\mathrm{I}, \mathrm{II}}$ are the Fisher matrices computed from the waveforms $\tilde{h}_{\mathrm{I}, \mathrm{II}}(f)$. The parameters used will be the ones listed in Eq. (2.5), so that $\Gamma$ is a $9 \times 9$ matrix. However, the errors in the estimation of $\mu_{\mathrm{S}}$ and $\phi_{\mathrm{S}}$ obtained in this way will be converted to a solid angle $\Delta \Omega_{\mathrm{S}}$ centered around the actual source direction. Following the notation of [28],

$$
\Delta \Omega_{\mathrm{S}}=2 \pi \sqrt{\left(\Delta \mu_{\mathrm{S}} \Delta \phi_{\mathrm{S}}\right)^{2}-\left\langle\delta \mu_{\mathrm{S}} \delta \phi_{\mathrm{S}}\right\rangle^{2}},
$$

where the second term is the covariance between $\mu_{\mathrm{S}}$ and $\phi_{\mathrm{S}}$. A similar quantity $\Delta \Omega_{\mathrm{L}}$ is used to quantify the error estimate in the orientation of the binary's orbit.

In what follows, whenever it is necessary to consider a specific cosmological model we will assume a flat universe with Hubble constant $H_{0}=75 \mathrm{~km} \mathrm{~s}^{-1} \mathrm{Mpc}^{-1}$, matter density $\Omega_{\mathrm{m}}=0.27$, and dark energy density $\Omega_{\mathrm{d}}=0.73$, with $\Omega_{\text {Total }}=\Omega_{\mathrm{m}}+\Omega_{\mathrm{d}}=1$.

\section{THE RESULTS AND THEIR ASTROPHYSICAL IMPLICATIONS}

In this section we will discuss the extent to which LISA will be able to constrain cosmological parameters by observing a binary SMBH with a large signal-to-noise ratio (SNR) and measuring their parameters, most importantly their location on the sky and the luminosity distance. As we shall see, the number of clusters in LISA's angular error

\footnotetext{
${ }^{4}$ This is a different notation from Ref. [26], where the source angles measured in the fixed barycenter frame are denoted by $\left(\bar{\theta}_{\mathrm{S}}, \bar{\phi}_{\mathrm{S}}, \bar{\theta}_{\mathrm{L}}, \bar{\phi}_{\mathrm{L}}\right)$.
}

box reduces dramatically when using the FWF as compared to RWF, thereby enabling us to identify the host galaxy, or galaxy cluster. Consequently, it should be possible to measure the dark energy equation of state by combining LISA observations with electromagnetic observations.

We start by outlining the generic features of parameter estimation with higher harmonics; after that, we will focus on angular resolution and the estimation of luminosity distance, and what these can tell us about cosmology.

\section{A. Parameter estimation with the full waveform: General trends}

Inclusion of higher harmonics results in a significant improvement in the determination of a binary's parameters in the context of LISA, as is the case for ground-based detectors [20]. A typical variation of parameter estimation (PE) accuracy with PN orders in amplitude is displayed ${ }^{5}$ in Table I. We observe the following general features of amplitude-corrected waveforms with regard to PE: For all masses and all angles we have explored, there is a significant improvement in the estimation of all parameters for LISA when considering the full waveform as compared to the restricted PN waveform. The orbital frequency and the inspiral rate, and therefore the phase evolution of the waveform, are determined primarily by the chirp mass $\mathcal{M}$. Thus, accurate phase tracking leads to a precise measurement of $\mathcal{M}$. The phase also depends on the "mass difference" $\delta$ which, therefore, can also be measured quite accurately. This is borne out by Table I where the trend is shown to be true for the FWF also. Finally, note the spectacular improvement in angular resolution and the determination of luminosity distance in going from RWF to FWF, which will be the focus of the rest of this section.

We relegate to Appendix B a more critical and in-depth discussion of the trends observed with increasing PN order in amplitude and consequent inclusion of higher harmonics in the waveform.

\section{B. Effect of higher harmonics on angular resolution and luminosity distance}

Table II lists the one-sigma errors in parameters of interest for seven different combinations of the angular parameters, as in Ref. [26], each for three different binary masses. The angular parameters (cf. A1, .., A7 in the table) chosen are a coarse sample of the possible orientations of the orbit and the source's sky location, and our choice of masses is indicative of the different binary SMBH coalescences LISA is likely to observe with a large SNR. To make direct contact with astrophysical systems, we give the physical masses $M=M_{\text {phys }}$ and not the ob-

\footnotetext{
${ }^{5}$ The numerical values in all our tables and results are unaffected by the missing terms discussed in [25] to the accuracies quoted.
} 
TABLE I. Variation of parameter estimation errors with post-Newtonian orders in amplitude for a $\left(10^{6}, 10^{7}\right) M_{\odot}$ binary at $z=0.55$ (corresponding to a luminosity distance of $D_{L}=3 \mathrm{Gpc}$ for a Hubble constant $H_{0}=75 \mathrm{~km} \mathrm{~s}^{-1} \mathrm{Mpc}$, matter density $\Omega_{\mathrm{m}}=0.27$, and dark energy density $\Omega_{\mathrm{d}}=0.73$, with $\Omega_{\text {Total }}=\Omega_{\mathrm{m}}+\Omega_{\mathrm{d}}=1$ ). The angles are chosen to be $\mu_{S}=-0.8, \phi_{S}=1, \mu_{L}=0.5, \phi_{L}=3$.

\begin{tabular}{|c|c|c|c|c|c|c|c|c|}
\hline PN order & SNR & $\Delta \ln \mathcal{M}\left(10^{-6}\right)$ & $\begin{array}{l}\left(10^{6}, 10^{7}\right) M \\
\Delta \delta\left(10^{-6}\right)\end{array}$ & $\begin{array}{c}; z=0.55 \\
\Delta t_{\mathrm{C}}(\mathrm{sec})\end{array}$ & $\begin{array}{r}\mu_{\mathrm{S}}=-0.8 \\
\Delta \phi_{\mathrm{C}}(\mathrm{rad})\end{array}$ & $\begin{array}{c}\mathrm{S}=1, \mu_{\mathrm{L}}=0.5 \\
\Delta \ln D_{\mathrm{L}}\left(10^{-3}\right)\end{array}$ & $\begin{array}{l}\phi_{\mathrm{L}}=3 \\
\Delta \Omega_{\mathrm{S}}\left(10^{-5} \mathrm{str}\right)\end{array}$ & $\Delta \Omega_{\mathrm{L}}\left(10^{-5} \mathrm{str}\right)$ \\
\hline 0 & 1824 & 380 & 310 & 90 & 65 & 32 & 2400 & 6600 \\
\hline 0.5 & 2005 & 110 & 110 & 32 & 2.6 & 2.3 & 6.3 & 9.9 \\
\hline 1 & 1793 & 87 & 93 & 28 & 2.6 & 2.5 & 6.1 & 8.0 \\
\hline 1.5 & 1680 & 87 & 95 & 29 & 2.9 & 2.7 & 6.8 & 8.7 \\
\hline 2 & 1585 & 94 & 100 & 31 & 3.1 & 2.8 & 7.8 & 10 \\
\hline 2.5 & 1549 & 96 & 100 & 31 & 3.2 & 2.9 & 8.2 & 11 \\
\hline
\end{tabular}

served (i.e., redshifted) masses $M_{\mathrm{obs}}$. The two are related by $M_{\text {obs }}=(1+z) M_{\text {phys }}$, where $z$ is the cosmological redshift of the source. In order to compute the upper frequency cutoff, one should first convert the total physical mass to the total observed mass and then use the formula for the LSO frequency. Our sources are all at $z \simeq 0.55$, i.e., a luminosity distance of $D_{\mathrm{L}}=3 \mathrm{Gpc}$. Theoretical predictions of event rates for SMBH coalescence vary over a wide range, but the rate could be as high as 1 per year within $z=0.55$ [33]. (See Sec. IV for a discussion of our results for merger events occurring at a higher redshift.) The physical masses and the corresponding LSO frequencies in the form $\left(m_{1} / M_{\odot}, m_{2} / M_{\odot}, 7 F_{\mathrm{LSO}} / \mathrm{mHz}\right)$ are $\left(10^{5}\right.$, $\left.10^{6}, 9.03\right),\left(6.45 \times 10^{4}, 1.29 \times 10^{6}, 7.33\right)$ and $\left(10^{6}, 10^{7}\right.$, 0.903). Thus, the highest harmonic (at seven times the orbital frequency) of the heaviest system that we consider does not quite reach the sweet spot of LISA's sensitivity. For the other two systems the dominant harmonic is close to the sweet spot and higher harmonics sweep through LISA's sensitivity bandwidth.

The table lists the 1-sigma errors incurred in the estimation of all the parameters except for the errors on $\mu_{\mathrm{L}}, \phi_{\mathrm{L}}$ (the direction of orbital angular momentum), and $\phi_{\mathrm{C}}$. As usual, we have converted the error in the estimation of $\mu_{\mathrm{S}}$ and $\phi_{\mathrm{S}}$ to an error in the solid angle $\Delta \Omega_{\mathrm{S}}$ centered around the actual source direction. For the sake of completeness we have given, in alternate rows, the errors for both the RWF and FWF.

For certain values of the angular parameters the presence of the harmonics seems to have a considerable impact on the determination of the luminosity distance and the angular position of the source. The errors in the luminosity distance (i.e., $\Delta D_{\mathrm{L}}$ ) and the source's sky position (i.e., $\Delta \Omega_{\mathrm{S}}$ ) are reduced by factors up to 600 and 400 , respectively, while using FWF as compared to RWF. This means that the error box could be smaller by a factor of $2.4 \times 10^{5}$. However, what is relevant for cosmological applications is by how much the error in the sky position goes down (i.e., about a factor of 2.5 to 400). Interestingly, the heavier, and astrophysically most relevant, system of $\left(10^{6}, 10^{7}\right) M_{\odot}$, where none of the harmonics get close to the detector's sweet spot, shows the largest improvement in distance estimation and angular resolution in going from restricted to amplitude-corrected waveforms. This observation, based only on the few systems studied in this paper, is found to be generally true in an independent and more exhaustive study by Trias and Sintes [31]. The larger improvement over the RWF is to be expected since for very massive systems only the higher harmonics radiate significantly within the detector's bandwidth. However, what is striking is that, when considering only FWF, for most choices of angles the errors on distance and the angular resolution are almost at the same level as for lighter systems. Doppler modulation does not seem to affect the accuracy of estimation of parameters for the systems considered in this paper; they are expected to be important for systems with lower masses [26]. An alternative method to study these issues is by direct use of the three time delay interferometry variables. Work along these lines is in progress [34] and should provide an independent check of our results in the near future.

\section{Number of clusters in LISA's error box}

Binary black holes are standard sirens [5]. The amplitude of gravitational waves from a binary SMBH is proportional to $\mathcal{M}^{5 / 6} / D_{\mathrm{L}}$. As evidenced by the numbers in Table II, LISA will measure both the chirp mass $\mathcal{M}$ of the source and the amplitude of gravitational waves to a great precision. Thus, the luminosity distance to a source can be extracted by gravitational-wave observations alone. In order to derive the luminosity distance-redshift relation, it is also necessary to measure the redshift $z$ to the source, but LISA cannot measure $z$. However, it might be possible to determine the source's redshift if the host galaxy, or galaxy cluster, can be optically identified. Whether or not this is possible depends on how good LISA's angular resolution is, and whether it is small enough that no more than a few galaxies or galaxy clusters are found within the angular error box. ${ }^{6}$

To determine the number of galaxy clusters within a solid angle $\Delta \Omega_{\mathrm{S}}$ of the angular error box, we need the

\footnotetext{
${ }^{6}$ Note that we have to use the angular error box and cannot use the smaller volume error box also fixed by the luminosity distance. In order to do precision cosmology we have to measure the source's redshift independently of the luminosity distance.
} 
TABLE II. Accuracy in LISA's measurement of the various parameters in Eq. (2.5), for seven different sets of the angular parameters and three different combinations of the (physical) masses at a distance of $3 \mathrm{Gpc}(z=0.55)$. When the number of clusters in the error box on the sky is significantly larger than 1, it will not be possible to determine redshift unless the inspiral event has a clear optical counterpart; we have chosen not to quote results for $\Delta w$ in such cases. (Note that the error on $w$ is ultimately determined by both LISA's statistical errors and weak lensing errors in the determination of luminosity distance.) The figures clearly demonstrate significant improvement in parameter estimation when higher order terms are included.

\begin{tabular}{|c|c|c|c|c|c|c|c|c|c|c|c|c|c|}
\hline Orientation & $\mu_{\mathrm{S}}$ & $\begin{array}{c}\varphi_{\mathrm{S}} \\
(\mathrm{rad})\end{array}$ & $\mu_{\mathrm{L}}$ & $\begin{array}{c}\varphi_{\mathrm{L}} \\
(\mathrm{rad})\end{array}$ & Model & SNR & $\begin{array}{c}\Delta \ln D_{\mathrm{L}} \\
\left(10^{-2}\right)\end{array}$ & $\begin{array}{c}\Delta \Omega_{\mathrm{S}} \\
\left(10^{-6} \mathrm{str}\right)\end{array}$ & $\begin{array}{c}\Delta \ln \mathcal{M} \\
\left(10^{-6}\right)\end{array}$ & $\begin{array}{c}\Delta \delta \\
\left(10^{-6}\right)\end{array}$ & $\begin{array}{c}\Delta t_{\mathrm{C}} \\
(\mathrm{sec})\end{array}$ & $\mathbf{N}_{\text {clusters }}$ & $\Delta w$ \\
\hline \multicolumn{14}{|c|}{$\left(m_{1}, m_{2}\right)=\left(10^{5}, 10^{6}\right) M_{\odot}$} \\
\hline \multirow[t]{2}{*}{ A1 } & 0.3 & 5 & 0.8 & 2 & RWF & 750 & 1.2 & 12 & 6.0 & 31 & 1.7 & 0.25 & 0.068 \\
\hline & & & & & FWF & 754 & 0.88 & 4.3 & 4.6 & 23 & 1.2 & 0.088 & 0.050 \\
\hline \multirow[t]{2}{*}{$\mathrm{A} 2$} & -0.1 & 2 & -0.2 & 4 & RWF & 1168 & 1.1 & 110 & 4.7 & 21 & 1.7 & 2.2 & 0.062 \\
\hline & & & & & FWF & 1150 & 0.58 & 13 & 3.5 & 16 & 1.1 & 0.27 & 0.033 \\
\hline \multirow[t]{2}{*}{ A 3} & -0.8 & 1 & 0.5 & 3 & RWF & 2722 & 0.25 & 170 & 3.3 & 12 & 2.6 & 3.5 & $\cdots$ \\
\hline & & & & & FWF & 2497 & 0.17 & 26 & 2.7 & 9.7 & 1.1 & 0.53 & 0.0096 \\
\hline \multirow[t]{2}{*}{ A4 } & -0.5 & 3 & -0.6 & -2 & RWF & 1868 & 0.74 & 150 & 3.1 & 15 & 1.2 & 3.1 & $\ldots$ \\
\hline & & & & & FWF & 1781 & 0.19 & 13 & 2.5 & 12 & 0.58 & 0.27 & 0.011 \\
\hline \multirow[t]{2}{*}{ A5 } & 0.9 & 2 & -0.8 & 5 & RWF & 3740 & 15 & 84 & 2.3 & 8.0 & 2.1 & 1.7 & 0.82 \\
\hline & & & & & FWF & 2857 & 0.11 & 8.1 & 1.7 & 7.9 & 0.69 & 0.17 & 0.0062 \\
\hline \multirow[t]{2}{*}{ A6 } & -0.6 & 1 & 0.2 & 3 & RWF & 2185 & 0.42 & 220 & 3.9 & 15 & 2.9 & 4.5 & $\ldots$ \\
\hline & & & & & FWF & 2108 & 0.24 & 65 & 3.0 & 11 & 1.6 & 1.3 & 0.014 \\
\hline \multirow[t]{2}{*}{ A7 } & -0.1 & 3 & -0.9 & 6 & RWF & 2213 & 0.58 & 410 & 3.5 & 13 & 1.1 & 8.4 & $\cdots$ \\
\hline & & & & & FWF & 2175 & 0.45 & 300 & 2.9 & 10 & 0.74 & 6.1 & $\ldots$ \\
\hline \multicolumn{14}{|c|}{$\left(m_{1}, m_{2}\right)=\left(6.45 \times 10^{4}, 1.29 \times 10^{6}\right) M_{\odot}$} \\
\hline \multirow[t]{2}{*}{ A1 } & 0.3 & 5 & 0.8 & 2 & RWF & 385 & 1.3 & 21 & 5.5 & 13 & 3.2 & 0.43 & 0.073 \\
\hline & & & & & FWF & 511 & 1.0 & 8.4 & 4.2 & 9.1 & 2.1 & 0.17 & 0.056 \\
\hline \multirow[t]{2}{*}{ A 2} & -0.1 & 2 & -0.2 & 4 & RWF & 595 & 1.1 & 120 & 4.2 & 9.2 & 2.5 & 2.4 & 0.062 \\
\hline & & & & & FWF & 771 & 0.70 & 25 & 3.3 & 6.5 & 1.7 & 0.51 & 0.039 \\
\hline \multirow[t]{2}{*}{ A 3} & -0.8 & 1 & 0.5 & 3 & RWF & 1345 & 0.33 & 170 & 3.4 & 5.8 & 2.7 & 3.5 & $\ldots$ \\
\hline & & & & & FWF & 1573 & 0.25 & 53 & 2.6 & 4.2 & 1.6 & 1.1 & 0.014 \\
\hline \multirow[t]{2}{*}{ A4 } & -0.5 & 3 & -0.6 & -2 & RWF & 924 & 0.78 & 160 & 3.0 & 6.8 & 1.7 & 3.3 & $\ldots$ \\
\hline & & & & & FWF & 1158 & 0.26 & 27 & 2.3 & 5.0 & 1.0 & 0.55 & 0.015 \\
\hline \multirow[t]{2}{*}{ A5 } & 0.9 & 2 & -0.8 & 5 & RWF & 1863 & 15 & 87 & 2.4 & 3.8 & 2.2 & 1.8 & 1.0 \\
\hline & & & & & FWF & 1506 & 0.19 & 25 & 2.0 & 3.9 & 1.3 & 0.51 & 0.011 \\
\hline \multirow[t]{2}{*}{ A6 } & -0.6 & 1 & 0.2 & 3 & RWF & 1069 & 0.47 & 240 & 4.1 & 7.2 & 3.1 & 4.9 & $\ldots$ \\
\hline & & & & & FWF & 1378 & 0.32 & 110 & 2.9 & 4.8 & 2.1 & 2.2 & 0.018 \\
\hline \multirow[t]{2}{*}{ A7 } & -0.1 & 3 & -0.9 & 6 & RWF & 1093 & 0.57 & 420 & 3.1 & 6.1 & 1.6 & 8.6 & $\ldots$ \\
\hline & & & & & FWF & 1448 & 0.50 & 350 & 2.5 & 4.2 & 1.1 & 7.1 & $\cdots$ \\
\hline \multicolumn{14}{|c|}{$\left(m_{1}, m_{2}\right)=\left(10^{6}, 10^{7}\right) M_{\odot}$} \\
\hline \multirow[t]{2}{*}{ A1 } & 0.3 & 5 & 0.8 & 2 & RWF & 495 & 11 & 600 & 1400 & 1100 & 290 & 12 & $\ldots$ \\
\hline & & & & & FWF & 444 & 2.2 & 16 & 190 & 240 & 75 & 0.33 & 0.12 \\
\hline \multirow[t]{2}{*}{$\mathrm{A} 2$} & -0.1 & 2 & -0.2 & 4 & RWF & 773 & 10 & 6500 & 870 & 710 & 190 & 130 & $\ldots$ \\
\hline & & & & & FWF & 685 & 1.2 & 43 & 130 & 160 & 51 & 0.88 & 0.068 \\
\hline A 3 & -0.8 & 1 & 0.5 & 3 & RWF & 1824 & 3.2 & 24000 & 380 & 310 & 90 & 490 & $\cdots$ \\
\hline & & & & & FWF & 1549 & 0.29 & 82 & 96 & 100 & 31 & 1.7 & 0.016 \\
\hline A4 & -0.5 & 3 & -0.6 & -2 & RWF & 1249 & 6.9 & 2400 & 550 & 450 & 120 & 49 & $\ldots$ \\
\hline & & & & & FWF & 1081 & 0.34 & 40 & 110 & 130 & 40 & 0.82 & 0.019 \\
\hline A5 & 0.9 & 2 & -0.8 & 5 & RWF & 2493 & 110 & 8300 & 270 & 220 & 63 & 170 & $\ldots$ \\
\hline & & & & & FWF & 1954 & 0.18 & 18 & 200 & 180 & 49 & 0.37 & 0.010 \\
\hline A6 & -0.6 & 1 & 0.2 & 3 & RWF & 1465 & 4.7 & 53000 & 470 & 380 & 110 & 1100 & $\ldots$ \\
\hline & & & & & FWF & 1273 & 0.44 & 300 & 105 & 115 & 36 & 6.1 & $\cdots$ \\
\hline A7 & -0.1 & 3 & -0.9 & 6 & RWF & 1480 & 21 & 170000 & 520 & 390 & 98 & 3500 & $\cdots$ \\
\hline & & & & & FWF & 1300 & 1.3 & 3400 & 87 & 100 & 30 & 69 & $\ldots$ \\
\hline
\end{tabular}


comoving volume corresponding to a cone defined by $\Delta \Omega_{\mathrm{S}}$ whose height is the physical distance from the detector to the source, which, of course, depends on the cosmological model. In a universe whose matter density is $\Omega_{\mathrm{m}}$ and in which dark energy density takes the form of a cosmological constant ${ }^{7} \Omega_{\mathrm{d}}$ (with $\Omega_{\text {Total }}=\Omega_{\mathrm{m}}+\Omega_{\mathrm{d}}=1$ ), the comoving volume per unit redshift within a box of angular size $\Delta \Omega_{\mathrm{S}}$ is

$$
\frac{d V_{\mathrm{C}}(z)}{d z}=\frac{\Delta \Omega_{\mathrm{S}}}{H_{0}} \frac{D_{\mathrm{L}}^{2}(z)}{(1+z)^{2}} \frac{1}{\sqrt{\Omega_{\mathrm{m}}(1+z)^{3}+\Omega_{\mathrm{d}}}} .
$$

In the above, $D_{\mathrm{L}}(z)$, the luminosity distance as a function of redshift, is given by

$$
D_{\mathrm{L}}(z)=\frac{1+z}{H_{0}} \int_{0}^{z} \frac{d z^{\prime}}{\sqrt{\Omega_{\mathrm{m}}\left(1+z^{\prime}\right)^{3}+\Omega_{\mathrm{d}}}},
$$

where $H_{0}$ is the Hubble parameter at the current epoch. The comoving volume from the observer to the source within a cone defined by $\Delta \Omega_{\mathrm{S}}$ is simply the integral of Eq. (3.1):

$$
V_{\mathrm{C}}(z)=\int_{0}^{z} d z^{\prime} \frac{\Delta \Omega_{\mathrm{S}}}{H_{0}} \frac{D_{\mathrm{L}}^{2}\left(z^{\prime}\right)}{\left(1+z^{\prime}\right)^{2}} \frac{1}{\sqrt{\Omega_{\mathrm{m}}\left(1+z^{\prime}\right)^{3}+\Omega_{\mathrm{d}}}} .
$$

The number density of clusters at high redshifts is not known very well. Following Ref. [35] we assume that the number density of clusters is $\sim 2 \times 10^{-5} h^{3} \mathrm{Mpc}^{-3}$, where $h$ is the present value of the Hubble parameter in units of $100 \mathrm{~km} \mathrm{~s}^{-1} \mathrm{Mpc}^{-1}$. We take $h=0.75, \Omega_{\mathrm{m}}=0.27$, and $\Omega_{\mathrm{d}}=0.73$. For this choice of cosmological parameters the number of expected clusters within the LISA angular error box is given in the second to last column of Table II. Clearly, in many cases the number of clusters is of order 1 , which means that LISA will help us to uniquely identify the host galaxy cluster of a binary SMBH merger event within a redshift of $z=0.55$.

There is a caveat with regard to the number of clusters found within the error box that is important to mention at this stage. Note that we integrated the comoving volume up to $z=0.55$, the location of our source. In reality, we would not know the redshift of the source and are not fully justified in integrating only up to this point; in principle, we should consider all clusters within the error box up to much larger redshifts. However, sources at redshifts much larger than $z=0.55$ for the same luminosity distance would probably give radically different cosmological parameters. Consistency with other observations justifies considering only galaxy clusters that are roughly in the redshift region determined by inverting a luminosity distance-redshift relation based on parameter values from

\footnotetext{
${ }^{7}$ In the next subsection we will relax this assumption.
}

other measurements. In any case, we have checked that integrating the comoving volume up to $z=1$ (which for our chosen value of $D_{\mathrm{L}}$ would already imply a very significant departure from current cosmological models) does not drastically change the results of Table II. Indeed, despite the higher limit of integration, the number of clusters in the angular error box remains less than 3 for most choices of angles, which is the (arbitrary) cutoff in $\mathbf{N}_{\text {clusters }}$ we have chosen to assess whether redshift can be determined.

There have been suggestions that in order to identify the source associated with a binary SMBH merger we should also look for optical/UV counterparts; the improved angular resolution with the correct signal model should help in this case too. Optical and other electromagnetic telescopes will need to survey a much smaller area on the sky than was thought before and should therefore more easily identify the galaxy cluster in which the merger took place.

The error in $D_{\mathrm{L}}$ being less than a percent means that we should, in principle, be able to tightly constrain the cosmological model a lot better. However, as discussed by several groups, the possible effect of weak gravitational lensing on parameter estimation, in particular, on our ability to measure the luminosity distance (see, e.g., Ref. [3]), will limit the extent to which LISA can measure dark energy. These considerations do not alter the main conclusions of this paper, as our main goal is to show that the field of view in LISA's angular resolution in most cases involves only a few sources.

\section{Constraints on the equation of state of dark energy}

We conclude by mentioning the implications of our estimates for astrophysics and cosmology. Up to this point in our analysis we have not assumed any electromagnetic counterpart to the coalescence events LISA will observe. But in reality, many of the LISA observations are likely to have electromagnetic counterparts, either as a precursor or as an afterglow [36,37]. The implications of a uniquely identified quasar source in coincidence with LISA were examined in [38] specifically assuming quasars as a possible electromagnetic counterpart. Even a single event of this type would provide us with unprecedented tests of SMBH accretion physics, such as a precise measurement of the Eddington ratio [38]. For this to be possible, and to identify a unique electromagnetic event in coincidence with a merger event as seen by LISA, the use of higher harmonics would be crucial, since for many of the directions in the sky it brings down the number of candidate clusters to less than 1 .

Another exciting possibility is to use LISA as a cosmological probe. If a unique host is identified electromagnetically in coincidence with the LISA observation, the redshift of the host galaxy will be known to very high accuracy. The improved estimate of the luminosity distance obtained by using the FWF would play a crucial 
role in determining the cosmological parameters as suggested by Schutz [5].

Gravitational-wave observation of a single inspiral event coupled with an electromagnetic determination of the redshift would allow LISA to strongly constrain the equation of state of dark energy. In a flat universe, the luminosity distance can be written as

$$
D_{\mathrm{L}}=(1+z) \int_{0}^{z} \frac{d z^{\prime}}{H\left(z^{\prime}\right)},
$$

where $H(z)$ is the Hubble parameter. Given a form of matter energy with density parameter $\Omega_{\mathrm{d}}$ and a (constant) equation-of-state index $w$, one has

$$
H(z)=H_{0}\left[\Omega_{\mathrm{m}}(1+z)^{3}+\Omega_{\mathrm{d}}(1+z)^{3(1+w)}\right]^{1 / 2} .
$$

For a given, fixed redshift (and fixed $H_{0}, \Omega_{\mathrm{m}}$, and $\Omega_{\mathrm{d}}$ ), the error on $w$ is

$$
\Delta w=D_{\mathrm{L}}\left|\frac{\partial D_{\mathrm{L}}}{\partial w}\right|^{-1} \frac{\Delta D_{\mathrm{L}}}{D_{\mathrm{L}}} .
$$

Using (3.4) and (3.5) and setting $\Omega_{\mathrm{d}}=0.73, w=-1$, and $D_{\mathrm{L}}=3 \mathrm{Gpc}$, one obtains

$$
\left|\frac{\partial D_{\mathrm{L}}}{\partial w}\right| \simeq 533 \mathrm{Mpc} .
$$

With these assumptions and using the values for $\Delta D_{\mathrm{L}} / D_{\mathrm{L}}$ from our analysis, we find that, in all of our examples, the FWF always leads to a smaller value for $\Delta w$ than the RWF whenever a comparison can be made. Indeed, in Table II there are many instances where the RWF does not allow for a determination of the redshift because the number of clusters in the angular error box on the sky is too large, in which case $w$ cannot be measured. In most of the examples we have considered, the FWF does not have this problem.

The foregoing analysis does not take into account the error in luminosity distance arising as a result of weak lensing of gravitational waves by the intervening mass concentrations between the binary SMBH source and LISA. It is estimated that the weak lensing will introduce errors in the luminosity distance at the level of about 3\%$5 \%$ for sources at $z \sim 0.5$ [3]. This is far greater than the systematic error in LISA's measurement of the luminosity distance. Therefore, weak lensing will be the limiting factor in LISA's ability to measure the dark energy equation of state unless weak lensing can be corrected by properly modeling the weak lenses.

We now revisit the caveat in the previous subsection regarding the fact that we tend to consider an error cone that stretches only to $z=0.55$. Let us evaluate $\Delta w$ when considering a measured luminosity distance of $D_{\mathrm{L}}=$ $3 \mathrm{Gpc}$, but allowing for the possibility that the source may be at a different redshift. Given our luminosity distance, to assume that the source could be at, e.g., $z=0.6$ and keeping $H_{0}, \Omega_{\mathrm{m}}$, and $\Omega_{\mathrm{d}}$ the same would already require $w=-0.47$ [from Eqs. (3.4) and (3.5)], a value excluded by WMAP and SNLS results [2]. In that case $\left|\partial D_{\mathrm{L}} / \partial w\right| \simeq 668 \mathrm{Mpc}$; substituting this into the right-hand side of Eq. (3.6) one finds that all values of $\Delta w$ will decrease by about $25 \%$ compared with the $z=0.55(w=$ -1) case. In reality, uncertainties in $H_{0}, \Omega_{\mathrm{m}}$, and $\Omega_{\mathrm{d}}$ will also have to be taken into account, but it will probably not be necessary to consider potential sources in the angular error box at redshifts that differ from the "favored" value by more than $20 \%$. A more complete treatment should take into account the covariances among $H_{0}, \Omega_{\mathrm{m}}, \Omega_{\mathrm{d}}$, and $w$; this we relegate to a future study.

We conclude by noting another interesting feature of our analysis. In addition to the improved angular resolution and luminosity distance, the errors in the estimation of mass parameters reduce considerably while using FWF. Even though the RWF itself would give a very good estimate of the masses, the improved measurement of the mass parameters would be very important in performing certain novel tests of general theory relativity and its alternatives $[7,39,40]$. For example, the use of FWF should help improve the accuracy with which the individual phasing coefficients can be determined independent of each other, an idea proposed in Refs. $[39,40]$.

\section{FACTORS AFFECTING THE ESTIMATES}

In this section we will briefly discuss some caveats regarding our results. Here we focus on physical issues; limitations related to our chosen methods for computing errors (and their resolution) are commented on in Appendix A.

(a) Effect of black hole spins. - Our analysis is restricted to the case of nonspinning black holes, while astrophysical black holes are known to have significant amounts of spin. Including the spin effect could significantly alter the estimation of different parameters [7]. To get an estimate of the effect of the spin on parameter estimation, we have recomputed the covariance matrix by including the leading order spin-orbit parameter in the waveform model. We observed a deterioration of up to an order of magnitude for the mass parameters but only a factor of a few in the estimation of luminosity distance and angular resolution. As suggested by Vecchio [41], the inclusion of precession should compensate for the deterioration of the mass errors to a great extent. For instance, including the leading spin-orbit term with a simple precession model, Ref. [41] showed that the angular resolution and luminosity distance determination could be improved by a factor between 3 and 10. A more recent analysis [42] incorporated the $2 \mathrm{PN}$ spin effects. Thus our estimates may not be too far from the realistic case despite the assumption of nonspinning holes. An interesting 
exercise for the future would be to include the amplitude corrections with the spin effects and see the extent to which the results of this paper change.

(b) Weak and strong gravitational lensing. - An important physical effect we have neglected in our analysis is the possibility of gravitational lensing of the signal. Weak lensing by intervening matter distributions distorts the gravitational waveform, inducing a systematic error in the estimation of luminosity distance [3]. This could be as high as 5\%-10\% for some of the systems, much higher than LISA's systematic error on the luminosity distance. On the other hand, strong lensing may improve the angular resolution, as multiple gravitational-wave "images" formed will reduce the correlations between various parameters [43]. This improvement could be as high as 100 times for a million solar mass (redshifted) binary [44]. Thus the net effect of lensing of a high redshift source may not be disastrous.

(c) Inaccuracy in the cosmological parameters. - In computing the accuracy with which we can measure the dark energy parameter $w$, we have assumed that other cosmological parameters, namely, $H_{0}, \Omega_{\mathrm{m}}$, and $\Omega_{\mathrm{d}}$, are all known precisely and that the redshift $z$ of the host galaxy is measured accurately. In reality, all these quantities will have their own statistical errors that must be folded into the analysis and we hope to do so in a future publication. Moreover, it might be possible for LISA to measure the luminosity distance-redshift curve by observing many supermassive black hole binary coalescences during its mission lifetime and extract all the cosmological parameters. How well LISA might be able to do would be a very interesting question that we would like to reserve for future studies. In any case, the fact that higher harmonics enable a greater accuracy in the measurement of the parameters should help.

(d) Event rates and measurement of $w$.- The rate of binary SMBH mergers in the Universe is not known with good accuracy. The rate depends on the cosmological model of choice and on structure formation and growth. Current models predict rates that vary over 2 orders of magnitude. For one of the models the rate of binary SMBH merger is as large as $\sim 10 \mathrm{yr}^{-1}$ within $z=1$ or about $\sim 1 \mathrm{yr}^{-1}$ up to $z=0.55$ (see [33] for an overview). A single source at the right point in parameter space at $z=0.55$ will be good enough to measure $w$ to a few percent. However, events at $z=1$ will be more frequent and consequently a few of them are likely to end up in the favored region of parameter space. We have verified that at $z=1$, for all choices of masses considered and angles A1-A5 (except for A3 in the case of the most massive system), the number of clusters within the error box is less than 10 when using the FWF. It should therefore be possible to either directly identify the merger source (by demanding consistency of the inferred cosmological parameters with those from other observations), or to locate it by observing an afterglow. Values of $\Delta w$ will be about $70 \%$ higher than before; however, with repeated observations one would be able to perform a statistical analysis along the lines of [5]. Thus, merger events at $z=1$ also allow for a measurement of $w$, although in this case the microlensing would probably restrict our accuracy in its estimation to $\sim 10$ percent [3].

\section{CONCLUSIONS}

In this paper we have reassessed LISA's ability to perform precision cosmology by using waveform models with far richer structure than those used before, and we find remarkable improvement in its angular resolution and estimation of the luminosity distance. Our ability to reliably measure the parameters of a binary SMBH depends crucially on the signal model onto which the data is projected. If the signal model is incorrect or even inaccurate, then regardless of how good the instrument might be, the measurement is prone to systematic errors which will make precision measurements meaningless (see, e.g., Ref. [45] for a detailed discussion on parameter extraction errors due to inaccurate template waveforms). From the point of view of incurring the minimum possible errors in the estimation of parameters, it is desirable to use the best known signal model. We have provided critical insight into the role of higher harmonics in the fast gravitational-wave phasing, higher PN corrections in the amplitudes, and their interplay with the slow modulations induced by LISA's motion (see Appendix B).

By using a waveform whose phase is correct to order $v^{7}$ (3.5PN) and with the amplitudes correct to order $v^{5}$ (2.5PN), we have found that LISA's angular resolution improves typically by a factor of 10 , even with a very conservative choice for the detector's lower cutoff frequency (i.e., $10^{-4} \mathrm{~Hz}$ ). This means that LISA will be able to uniquely identify the galaxy cluster in which the merger event took place and thereby facilitate optical identification and the measurement of the redshift of the source. Together with the fact that binary SMBH sources are standard sirens, this means that LISA will be able to measure the cosmological parameters by circumventing the lower rungs of the cosmic distance ladder.

\section{ACKNOWLEDGMENTS}

For useful discussions and encouragement we would like to thank Karsten Danzmann and Bernard Schutz. We benefited from discussions on the importance of weak lensing in the context of LISA with Daniel Eisenstein, 
Daniel Holz, and Scott Hughes. We are grateful to Miquel Trias and Alicia Sintes for sharing with us their results, which corroborate ours. B. R. Iyer thanks Cardiff University and IHES, France for hospitality during different stages of this work. This research was supported in part by PPARC Grant No. PP/B500731/1. The calculations leading to the results of this paper were performed with the aid of MATHEMATICA.

\section{APPENDIX A: IMPLEMENTATION OF THE PARAMETER ESTIMATION WITH FWF AND ISSUES RELATED TO NUMERICAL ACCURACY}

The computation of the covariance matrix for LISA using FWF starts with a simpler code (in MATHEMATICA) which reproduces the results of Refs. $[13,14,20]$ for the ground-based detectors. The modulations due to LISA's orbital motion are then accounted for to obtain the waveform in LISA's frame. This waveform is differentiated analytically and the derivatives are stored in an array and used in the computation of the various elements of the Fisher matrix. The integrations that are needed in computing different elements of the Fisher information matrix are performed using numerical integration routines of MATHEMATICA. The program thus evaluates the Fisher matrix for the given input values of the component masses of the binary, the four angles describing the source in the solar-system barycenter frame, and the PN order of the amplitude for a fixed distance of $3 \mathrm{Gpc}$ (phasing is always 3.5PN). The input masses are always physical masses consistent with the cosmological model we described earlier.

We have checked that our code reproduces the numbers in Ref. [26] (with the corresponding restricted waveform which uses a $1.5 \mathrm{PN}$ phasing including the spin-orbit parameters, and the corresponding noise PSD) with less than $1 \%$ difference. Since we use the noise PSD of Ref. [7], we have checked that we obtain the numbers for the patternaveraged case of [7] to less than $1 \%$ difference using their waveform parametrization, which includes all nonspinning and spinning binary parameters up to $2 \mathrm{PN}$ but ignores higher PN order terms in the phasing. Lastly, we recover the results of [27], which uses a 3.5PN phasing for the parameter estimation, and recover the results again to less than $1 \%$ difference.

The numbers quoted in this paper were checked with three independent codes, all in MATHEMATICA, but using different numerical integration routines (such as NINTEGRATE and LISTINTEGRATE) and matrix inversion routines [such as INVERSE and another method based on singular value decomposition (SVD)], and all three codes agree to less than $5 \%$.

A large dimension of the parameter space often leads to ill-conditioned Fisher matrices; i.e., the magnitude of the ratio of the smallest and the largest of its singular values (the inverse of the condition number) approaches the ma- chine's floating point precision $\left(10^{-16}\right.$ in our case). For the $\left(6.45 \times 10^{4}, 1.29 \times 10^{6}\right) M_{\odot}$ system, only the RWF Fisher matrix is ill-conditioned, for the third angle. The $\left(10^{5}, 10^{6}\right) M_{\odot}$ system, for both RWF and FWF, is free of ill-conditioned Fisher matrices. More specifically, the inverse of the condition number is always $10^{3}-10^{5}$ times larger than $10^{-16}$. The MATHEMATICA inversion routine as well as SVD are used to obtain the covariance matrix, and both methods give the same results. For these systems, we also note that the numerical eigenvalues (computed using the MATHEMATICA function EIGENVALUES) coincide with the numerical singular values (obtained through SINGULARVALUEDECOMPOSITION) at the standard floating point precision of $10^{-16}$. On the other hand, the Fisher matrices for the $\left(10^{6}, 10^{7}\right) M_{\odot}$ system show ill-conditioned behavior at this precision. However, this may not mean that the results obtained are unreliable for reasons outlined next. The SVD of the Fisher matrices shows that one or more of the singular values approach zero. Indeed, a machine precision calculation of the singular values yields zero for at least one singular value of the Fisher matrices for this system. In such cases, we replace the singular values by the numerical eigenvalues (whose machine precision calculation does not yield zero) to obtain the inverse using SVD. However, MATHEMATICA has the option of computing at higher precision by padding unknown digits beyond the known ones. Using this facility and repeating the inversion procedure using SVD but with precision higher than $10^{-16}$ does not alter the results and shows that the singular values are equal to the eigenvalues, as expected, with none of them being zero. The condition number is also seen to be 10 times larger than the precision used. In the case of one or more almost zero singular values (at standard floating point precision), one can also obtain a pseudoinverse [46] that is closest to the "real" inverse, in a least-squares sense, and end up with different results. However, for the $\left(10^{6}, 10^{7}\right) M_{\odot}$ system, the errors given by the pseudoinverse do not seem physically correct and so we refrain from using it in this work. A detailed analysis, by perturbing the Fisher matrices and observing how the inverses behave, will be a stronger test of the reliability of our results [47].

A recent Markov Chain Monte Carlo (MCMC) analysis of Ref. [48] revealed an interesting point relevant to our analysis. It compared the estimates from the Fisher information matrix with those obtained from MCMC and found that there is excellent agreement between the two methods in the case of the extrinsic parameters (what we refer to as $\mathbf{p}_{\text {slow }}$ ), although in the case of the intrinsic parameters the two methods are not in good agreement. This is good news since our main concern here is the angular resolution and the luminosity distance. Though their analysis used the restricted waveform, we believe that similar trends would exist in the case of FWF too since the dimensionality of the parameter space is the same as that of RWF. 


\section{APPENDIX B: PARAMETER ESTIMATION WITH THE FWF AND VARIATION WITH PN ORDER IN AMPLITUDE}

In this appendix we will attempt to provide some qualitative understanding of the trends observed in the parameter estimation accuracy with increasing $\mathrm{PN}$ order in amplitude. Recall that the covariance matrix goes as $1 / \mathrm{SNR}^{2}$ and therefore errors go down as $1 / \mathrm{SNR}$. However, notice from Table I that, even though the FWF SNR is less than the RWF SNR, all errors decrease as we go from RWF to FWF. This provides the clue that the SNR cannot account for the improved performance of FWF: In going from the RWF to $0.5 \mathrm{PN}$, the ratios of the two SNRs are nowhere close to the inverse ratios of the errors in various parameters. Further, as we go from $0.5 \mathrm{PN}$ to $2.5 \mathrm{PN}$, although the products of the SNR with errors do not fluctuate much, they are noticeably different, implying that the SNR is not the main reason why the parameter estimation improves.

Next, we note that at $0.5 \mathrm{PN}$ both the first and the third harmonic possess the same structure ( $f$ dependence) and almost the same variety (dependence on the inclination angle ı) (compare Eqs. (5.7b) and (5.8b) of [24]). However, their effects on PE, when examined independently, are vastly different, as seen in Table III. The 0.5PN PE is hardly distinguishable from the PE with the first harmonic suppressed, and thus the improvement at $0.5 \mathrm{PN}$ is solely due to the third harmonic. This is further confirmed by performing the PE with a "mock" FWF where the third harmonic is replaced by the second harmonic: as seen from Table III, the PE worsens in this case. Thus, the presence of higher harmonics brings about improved PE. It must, however, be pointed out that the improvement is obtained not merely due to the presence of the higher harmonic but crucially due to the increased span of the $k$ th harmonic in the frequency domain $\left[k\left(F_{\text {LSO }}-F_{\text {in }}\right)\right]$. This is first checked by working with a mock $0.5 \mathrm{PN}$ waveform containing the second harmonic but using for its span the increased span of the third. It is confirmed by an alternative mock waveform, suppressing the first and third harmonics and including instead the seventh harmonic at $0.5 \mathrm{PN}$. The dramatic improvement in $\mathrm{PE}$ compared to the regular 0.5PN waveform, but with almost the same SNR, shows that at the $0.5 \mathrm{PN}$ level, where complications due to PN corrections to the harmonics and higher order expansion coefficients of $\dot{F}^{-1 / 2}$ are not present, the SNR is not the determining factor.

To understand the variation in PE from 0.5 to $2.5 \mathrm{PN}$ we need to disentangle many effects. The FWF introduces more structure ( $f$ dependences) at different $\mathrm{PN}$ orders. Different harmonics appear at different PN orders and in our model are associated with their respective spans. At 2.5PN, higher order corrections appear in every harmonic except the sixth and the seventh harmonics. The frequency sweep $\dot{F}^{-1 / 2}$ in the Fourier transform of the binary signal brings in further PN corrections associated with it [the coefficients $S_{(m / 2)}$ in Eq. (2.3)]. Finally, the coefficients involve angles which are fixed in the barycentric frame but suffer modulations (Doppler and orientational) due to LISA's motion. To this end, we reexamined the PE with a series of mock waveforms including, where possible, only one of the above aspects. For instance, in Table IV we consider only the second harmonic and its associated PN corrections. From the results it is clear that although PN corrections to the second harmonic bring in additional terms, they do not improve PE; rather, in comparison with the RWF, the PE worsens. This is probably due to the fact that PN corrections with the same frequency dependence as before (or powers thereof) increase the

TABLE III. Parameter estimation at 0.5PN order with mock waveforms corresponding to different choices. The first (second) row corresponds to the restricted $(0.5 \mathrm{PN})$ results. The third (fourth) row corresponds to the 0.5PN waveform with only the third (first) harmonic and suppressing the first (third) harmonic. The fifth row corresponds to a mock waveform suppressing the first harmonic and replacing the third harmonic by the second. This implies retaining its polarization amplitude and phase but changing $t(f / 3) \rightarrow t(f / 2)$ in the Fourier and the Doppler phases and decreasing the frequency span from $3\left(F_{\mathrm{LSO}}-F\right.$ in $)$ to $2\left(F_{\mathrm{LSO}}-F_{\text {in }}\right)$. The sixth row corresponds to the previous case but with an enhanced span $3\left(F_{\text {LSO }}-F_{\text {in }}\right)$. The last row corresponds to a mock waveform where the seventh harmonic is moved from $2.5 \mathrm{PN}$ to $0.5 \mathrm{PN}$ and treated with its associated normal frequency span $7\left(F_{\mathrm{LSO}}-F_{\text {in }}\right)$ with the first and third harmonics suppressed. In this and other tables in this appendix the system and angles are the same as in Table I.

\begin{tabular}{|c|c|c|c|c|c|c|c|c|}
\hline PN order & SNR & $\begin{array}{r}\Delta \ln \mathcal{M} \\
\left(10^{-6}\right)\end{array}$ & $\begin{array}{c}\left.10^{7}\right) M_{\odot} \\
\Delta \delta \\
\left(10^{-6}\right)\end{array}$ & $\begin{array}{l}=0.55 \\
\Delta t_{\mathrm{C}} \\
(\mathrm{sec})\end{array}$ & $\begin{array}{c}=-0 . \\
\Delta \phi_{\mathrm{C}} \\
(\mathrm{rad})\end{array}$ & $\begin{array}{c}{ }_{\mathrm{S}}=1, \mu \\
\Delta \ln D_{\mathrm{L}} \\
\left(10^{-3}\right)\end{array}$ & $\begin{array}{c}0.5, \phi_{\mathrm{L}}=3 \\
\Delta \Omega_{\mathrm{S}} \\
\left(10^{-5} \mathrm{str}\right)\end{array}$ & $\begin{array}{l}\text { 3). } \\
\qquad \Omega_{\mathrm{L}} \\
\left(10^{-5} \mathrm{str}\right)\end{array}$ \\
\hline 0 & 1824 & 380 & 310 & 90 & 65 & 32 & 2400 & 6600 \\
\hline 0.5 & 2005 & 110 & 110 & 32 & 2.6 & 2.3 & 6.3 & 9.9 \\
\hline 0.5 (third) & 2004 & 110 & 110 & 32 & 2.6 & 2.3 & 6.3 & 10 \\
\hline 0.5 (first) & 1825 & 370 & 310 & 81 & 21 & 10 & 360 & 960 \\
\hline 0.5 (second) & 1648 & 410 & 340 & 90 & 12 & 10 & 690 & 1800 \\
\hline 0.5 (second with span of third) & 1757 & 190 & 140 & 31 & 5.0 & 2.2 & 17 & 44 \\
\hline 0.5 (seventh) & 1943 & 11 & 22 & 8.5 & 1.9 & 2.1 & 3.6 & 4.2 \\
\hline
\end{tabular}


TABLE IV. Variation of PE with PN orders in amplitude for a mock FWF retaining only the second harmonic and its higher order PN corrections. The PN corrections to a given harmonic at PN order $n / 2$ add terms of type $(2 \pi M f / k)^{n / 3}$. While they bring in new structure in the waveform, they do not help improve PE; instead, they enhance the covariances among different parameters and thereby worsen PE relative to RWF.

\begin{tabular}{|c|c|c|c|c|c|c|c|c|}
\hline PN order & SNR & $\begin{array}{r}\Delta \ln \mathcal{M} \\
\left(10^{-6}\right)\end{array}$ & $\begin{array}{c}\left(10^{6}, 10\right. \\
\Delta \delta \\
\left(10^{-6}\right)\end{array}$ & $\begin{array}{l}z=0 . \\
\Delta t_{\mathrm{C}} \\
(\mathrm{sec})\end{array}$ & $\begin{array}{l}=-0 \\
\Delta \phi_{\mathrm{C}} \\
(\mathrm{rad})\end{array}$ & $\begin{array}{r}=1, \mu_{\mathrm{L}} \\
\Delta \ln D_{\mathrm{L}} \\
\left(10^{-3}\right)\end{array}$ & $\begin{array}{c}\mathrm{b}_{\mathrm{L}}=3(\mathrm{~A} 3) . \\
\Delta \Omega_{\mathrm{S}} \\
\left(10^{-5} \mathrm{str}\right)\end{array}$ & $\begin{array}{c}\Delta \Omega_{\mathrm{L}} \\
\left(10^{-5} \mathrm{str}\right)\end{array}$ \\
\hline $0,0.5$ & 1824 & 380 & 310 & 90 & 65 & 32 & 2400 & 6600 \\
\hline $1,1.5$ & 1510 & 450 & 370 & 110 & 110 & 44 & 2000 & 5900 \\
\hline 2 & 1387 & 490 & 400 & 120 & 120 & 45 & 2300 & 6700 \\
\hline 2.5 & 1353 & 500 & 410 & 120 & 130 & 48 & 2400 & 7200 \\
\hline
\end{tabular}

covariances among the various parameters and thus worsen PE.

In Table $\mathrm{V}$, on the other hand, the mock waveform includes all the higher harmonics but excludes their PN amplitude corrections and PN corrections arising from the frequency sweep. Clearly, the PE improves monotonically as we go from one order to the next in all the parameters quoted, and so does the SNR. Additionally, an examination of the results in Tables $\mathrm{I}$ and $\mathrm{V}$ reveals that, while the higher harmonics by themselves tend to generically improve $\mathrm{PE}$, the $\mathrm{PN}$ corrections to the harmonics arising from the higher PN amplitudes and higher PN frequency sweep tend to degrade PE. However, their effect is less dramatic than one may naively expect. This is because higher harmonics necessarily appear at higher PN orders and, as is evident from Eq. (2.3), they come with higher powers of the PN expansion parameter: $\left(2 \pi M f_{k}\right)^{n / 3}$ for the $k$ th harmonic appearing at the $n / 2$ th PN order. The upper cutoff of the $k$ th harmonic in the frequency domain is $k F_{\text {LSO }}$ at which $(2 \pi M f)^{1 / 3}$ reaches its maximum of $6^{-1 / 2}$. The seventh harmonic, for example, will be scaled by a factor which is always less than $6^{-5 / 2}$ and consequently will

TABLE V. Variation of PE with PN orders for a mock FWF retaining all the harmonics and neglecting both higher order PN corrections to them and also PN corrections to $\left(\frac{d F}{d t}\right)^{-1 / 2}$ arising from the frequency sweep. Thus most higher order PN corrections to the harmonics are neglected. Higher harmonics generally improve parameter estimation. PN corrections to harmonics tend to degrade PE.

\begin{tabular}{|c|c|c|c|c|c|c|c|c|}
\hline PN order & SNR & $\begin{array}{r}\Delta \ln \mathcal{M} \\
\left(10^{-6}\right)\end{array}$ & $\begin{array}{c}\left(10^{6}, 10\right. \\
\Delta \delta \\
\left(10^{-6}\right)\end{array}$ & $\begin{array}{l}z=0 . \\
\Delta t_{\mathrm{C}} \\
(\mathrm{sec})\end{array}$ & $\begin{array}{l}=-0 \\
\Delta \phi_{\mathrm{C}} \\
(\mathrm{rad})\end{array}$ & $\begin{array}{r}=1, \mu_{\mathrm{L}} \\
\Delta \ln D_{\mathrm{L}} \\
\left(10^{-3}\right)\end{array}$ & $\begin{array}{c}\phi_{\mathrm{L}}=3(\mathrm{~A} 3) . \\
\Delta \Omega_{\mathrm{S}} \\
\left(10^{-5} \mathrm{str}\right)\end{array}$ & $\begin{array}{c}\Delta \Omega_{\mathrm{L}} \\
\left(10^{-5} \mathrm{str}\right)\end{array}$ \\
\hline 0 & 1824 & 380 & 310 & 90 & 65 & 32 & 2400 & 6600 \\
\hline 0.5 & 2005 & 110 & 110 & 32 & 2.6 & 2.3 & 6.3 & 9.9 \\
\hline 1 & 2058 & 83 & 87 & 26 & 2.4 & 2.2 & 5.1 & 7.03 \\
\hline 1.5 & 2068 & 78 & 82 & 25 & 2.3 & 2.2 & 4.8 & 6.4 \\
\hline 2 & 2070 & 77 & 82 & 24 & 2.3 & 2.2 & 4.8 & 6.3 \\
\hline 2.5 & 2070 & 77 & 82 & 24 & 2.3 & 2.2 & 4.8 & 6.3 \\
\hline
\end{tabular}

TABLE VI. Correlation coefficients computed using RWF for a binary at $z \simeq 0.55$ comprising $\left(10^{6}, 10^{7}\right) M_{\odot}$ SMBH. The table shows how the "fast" and "slow" variables behave. There is a high correlation among parameters in the same subclass, but only a weak correlation between members of different subclasses. Entries that are vacant can be found by symmetry.

\begin{tabular}{|c|c|c|c|c|c|c|c|c|c|}
\hline & & & $10^{6}, 10^{7}$ & orrelatior & ficient $\mathrm{m}$ & OPN & & & \\
\hline & $\ln \mathcal{M}$ & $\delta$ & $t_{\mathrm{C}}$ & $\phi_{\mathrm{C}}$ & $\ln D_{\mathrm{L}}$ & $\mu_{\mathrm{S}}$ & $\phi_{\mathrm{S}}$ & $\mu_{\mathrm{L}}$ & $\phi_{\mathrm{L}}$ \\
\hline $\ln \mathcal{M}$ & 1 & -0.99 & 0.93 & -0.19 & -0.17 & 0.14 & -0.13 & -0.15 & 0.031 \\
\hline$\delta$ & & 1 & -0.92 & 0.098 & 0.084 & -0.06 & 0.065 & 0.074 & -0.0088 \\
\hline$t_{\mathrm{C}}$ & & & 1 & -0.43 & -0.45 & 0.33 & -0.43 & -0.46 & -0.022 \\
\hline$\phi_{\mathrm{C}}$ & & & & 1 & 0.98 & -0.95 & 0.70 & 0.92 & -0.39 \\
\hline $\ln D_{\mathrm{L}}$ & & & & & 1 & -0.88 & 0.81 & 0.98 & -0.22 \\
\hline$\mu_{\mathrm{S}}$ & & & & & & 1 & -0.45 & -0.77 & 0.65 \\
\hline$\phi_{\mathrm{S}}$ & & & & & & & 1 & 0.92 & 0.38 \\
\hline$\mu_{\mathrm{L}}$ & & & & & & & & 1 & -0.016 \\
\hline$\phi_{\mathrm{L}}$ & & & & & & & & & 1 \\
\hline
\end{tabular}


TABLE VII. Correlation coefficients computed using FWF for a binary at $z \simeq 0.55$, comprising $\left(10^{6}, 10^{7}\right) M_{\odot}$ SMBH. The table clearly shows a more pronounced decoupling between the "fast" and "slow" variables at 2.5PN. There is a high correlation among elements of the same subclass but a lower correlation between the fast and slow subclasses.

\begin{tabular}{|c|c|c|c|c|c|c|c|c|c|}
\hline & & & $\left.0^{6}, 10^{7}\right) \Lambda$ & correlati & efficient 1 & ix; $2.5 \mathrm{PN}$ & & & \\
\hline & $\ln \mathcal{M}$ & $\delta$ & $t_{\mathrm{C}}$ & $\phi_{\mathrm{C}}$ & $\ln D_{\mathrm{L}}$ & $\mu_{\mathrm{S}}$ & $\phi_{\mathrm{S}}$ & $\mu_{\mathrm{L}}$ & $\phi_{\mathrm{L}}$ \\
\hline $\ln \mathcal{M}$ & 1 & -0.97 & 0.94 & 0.37 & -0.075 & -0.0069 & -0.10 & -0.12 & -0.097 \\
\hline$\delta$ & & 1 & -0.99 & -0.54 & 0.06 & 0.030 & 0.093 & 0.093 & 0.11 \\
\hline$t_{\mathrm{C}}$ & & & 1 & 0.63 & -0.10 & -0.016 & -0.14 & -0.13 & -0.14 \\
\hline$\phi_{\mathrm{C}}$ & & & & 1 & 0.051 & -0.17 & 0.015 & 0.16 & -0.18 \\
\hline $\ln D_{\mathrm{L}}$ & & & & & 1 & -0.13 & 0.90 & 0.71 & 0.47 \\
\hline$\mu_{\mathrm{S}}$ & & & & & & 1 & 0.036 & -0.56 & 0.68 \\
\hline$\phi_{\mathrm{S}}$ & & & & & & & 1 & 0.76 & 0.71 \\
\hline$\mu_{\mathrm{L}}$ & & & & & & & & 1 & 0.18 \\
\hline$\phi_{\mathrm{L}}$ & & & & & & & & & 1 \\
\hline
\end{tabular}

contribute less power. The seventh harmonic in the FWF at its regular PN order hardly leads to any improvement in PE. However, as remarked earlier in Table III, had it appeared at $0.5 \mathrm{PN}$ its impact would have been substantial. We have verified these trends for another angle and for a lower mass system of $\left(10^{5}, 10^{6}\right) M_{\odot}$; for the sake of brevity we leave out the details.

Our last comment relates to the decoupling between the fast variables $\mathbf{p}_{\text {fast }}$ and the slow variables $\mathbf{p}_{\text {slow }}$ in the case of the FWF. We have checked that in the case of the FWF the correlation coefficients are very small (of the order of $0.1-0.3$ ) between these two subsets of parameters (see Tables VI and VII). There exists, however, a high correlation among different parameters of the same type as in the case of RWF (see, e.g., Ref. [6]). Specifically, as in the RWF case [6], the distance estimation could be improved if the source is better localized in the sky.

It is worth recalling that in the parameter estimation problem we are not only limited by statistical errors due to noise but also by theoretical or systematic errors arising at any PN order due to the limited accuracy of the waveforms. Recently, Cutler and Vallisneri [45] have looked into this issue more critically. In the context of the present work, we would like to stress that, despite the fact that the largest improvement in PE arises from the third harmonic at $0.5 \mathrm{PN}$, the need to limit systematic errors mandates the use of the best available waveform, i.e., the one at $2.5 \mathrm{PN}$ in amplitude and 3.5PN in phase.
[1] P. J.E. Peebles and B. Ratra, Rev. Mod. Phys. 75, 559 (2003).

[2] D. N. Spergel et al. (WMAP), Astrophys. J. Suppl. Ser. 170, 377 (2007).

[3] D. E. Holz and S. A. Hughes, Astrophys. J. 629, 15 (2005).

[4] N. Dalal, D. E. Holz, S. A. Hughes, and B. Jain, Phys. Rev. D 74, 063006 (2006).

[5] B. F. Schutz, Nature (London) 323, 310 (1986).

[6] S. A. Hughes, Mon. Not. R. Astron. Soc. 331, 805 (2002).

[7] E. Berti, A. Buonanno, and C. M. Will, Phys. Rev. D 71, 084025 (2005).

[8] E. Berti, A. Buonanno, and C. M. Will, Classical Quantum Gravity 22, S943 (2005).

[9] L. Blanchet, T. Damour, B. R. Iyer, C. M. Will, and A. G. Wiseman, Phys. Rev. Lett. 74, 3515 (1995).

[10] L. Blanchet, Phys. Rev. D 54, 1417 (1996); 71, 129904(E) (2005).

[11] L. Blanchet, G. Faye, B. R. Iyer, and B. Joguet, Phys. Rev. D 65, 061501(R) (2002); 71, 129902(E) (2005).

[12] L. Blanchet, T. Damour, G. Esposito-Farèse, and B. R. Iyer, Phys. Rev. Lett. 93, 091101 (2004).
[13] C. Van Den Broeck, Classical Quantum Gravity 23, L51 (2006).

[14] C. Van Den Broeck and A. Sengupta, Classical Quantum Gravity 24, 155 (2007).

[15] K. G. Arun, B. R. Iyer, B. S. Sathyaprakash, and S. Sinha, Phys. Rev. D 75, 124002 (2007).

[16] A. M. Sintes and A. Vecchio, in Rencontres de Moriond: Gravitational Waves and Experimental Gravity, edited by J. Dumarchez (Frontières, Paris, 2000).

[17] A. M. Sintes and A. Vecchio, in Proceedings of the Third Amaldi Conference on Gravitational Waves, edited by S. Meshkov (AIP, New York, 2000), p. 403.

[18] T. A. Moore and R. W. Hellings, Phys. Rev. D 65, 062001 (2002).

[19] R.W. Hellings and T. A. Moore, Classical Quantum Gravity 20, S181 (2003).

[20] C. Van Den Broeck and A.S. Sengupta, Classical Quantum Gravity 24, 1089 (2007).

[21] T. Damour, B. R. Iyer, and B. S. Sathyaprakash, Phys. Rev. D 63, 044023 (2001); 72, 029902(E) (2005).

[22] T. Damour, B. R. Iyer, and B. S. Sathyaprakash, Phys. Rev. D 66, 027502 (2002); 66, 027502(E) (2002). 
[23] L. Blanchet, B. R. Iyer, C. M. Will, and A. G. Wiseman, Classical Quantum Gravity 13, 575 (1996).

[24] K. G. Arun, L. Blanchet, B. R. Iyer, and M. S. S. Qusailah, Classical Quantum Gravity 21, 3771 (2004); 22, 3115(E) (2005).

[25] L. E. Kidder, L. Blanchet, and B. R. Iyer, arXiv:0706.0726.

[26] C. Cutler, Phys. Rev. D 57, 7089 (1998).

[27] K. G. Arun, Phys. Rev. D 74, 024025 (2006).

[28] L. Barack and C. Cutler, Phys. Rev. D 69, 082005 (2004).

[29] L. Finn, Phys. Rev. D 46, 5236 (1992).

[30] L. Finn and D. Chernoff, Phys. Rev. D 47, 2198 (1993).

[31] M. Trias and A. M. Sintes, arXiv:0707.4434v1.

[32] B. Kocsis, Z. Haiman, K. Menou, and Z. Frei, Phys. Rev. D 76, 022003 (2007).

[33] See Chap. 3 of J. Baker et al., LISA Science Document LISA-LIST-RP-436, 2007, http://beyondeinstein.nasa. gov/education/beta/reading/LISA_Science_Case.pdf.

[34] S. Babak, A. Pai, and B. Schutz (unpublished).

[35] N. Bahcall et al., Astrophys. J. Suppl. Ser. 148, 243 (2003).

[36] M. Milosavljevic and E. S. Phinney, Astrophys. J. 622, L93 (2005).
[37] P. J. Armitage and P. Natarajan, Astrophys. J. 567, L9 (2002).

[38] B. Kocsis, Z. Frei, Z. Haiman, and K. Menou, Astrophys. J. 637, 27 (2006).

[39] K. G. Arun, B. R. Iyer, M.S.S. Qusailah, and B.S. Sathyaprakash, Classical Quantum Gravity 23, L37 (2006).

[40] K. G. Arun, B. R. Iyer, M.S.S. Qusailah, and B.S. Sathyaprakash, Phys. Rev. D 74, 024025 (2006).

[41] A. Vecchio, Phys. Rev. D 70, 042001 (2004).

[42] R. N. Lang and S. A. Hughes, Phys. Rev. D 74, 122001 (2006); 75, 089902(E) (2007).

[43] R. Takahashi and T. Nakamura, Astrophys. J. 595, 1039 (2003).

[44] N. Seto, Phys. Rev. D 69, 022002 (2004).

[45] C. Cutler and M. Vallisneri, arXiv:0707.2982.

[46] W. H. Press, S. A. Teukolsky, W. T. Vetterling, and B.P. Flannery, Numerical Recipes in Fortran (Cambridge University Press, Cambridge, England, 1992), 2nd ed.

[47] M. Vallisneri, arXiv:gr-qc/0703086.

[48] N. J. Cornish and E. K. Porter, Classical Quantum Gravity 23, S761 (2006). 